\title{
Pyrene-Assisted Synthesis of Size-Controlled Gold Nanoparticles in Sodium Dodecyl Sulfate Micelles
}

\author{
Jin-Pei Deng, ${ }^{\dagger}$ Chunhung Wu,$\stackrel{\dagger}{\dagger}$ Cheng-His Yang, ${ }^{\ddagger}$ and Chung-Yuan Mou*,† \\ Department of Chemistry, National Taiwan University, Taipei, Taiwan, and Department of \\ Chemistry, Tamkang University, Tamsui, Taiwan
}

Received May 11, 2005

\begin{abstract}
Gold nanoparticles prepared by chemical reduction in sodium dodecyl sulfate (SDS) solution are sizecontrolled with the addition of pyrene. Micellar electrokinetic capillary chromatography (MEKC) is applied to the system to examine the size and polydispersity of gold nanoparticles and to show that pyrene has the extraordinary effect in decreasing the size and narrowing the dispersity of gold nanoparticles. The MEKC electropherograms further suggest that pyrene could be oxidized by the aqueous Au(III) complexes first. All the reduced Au complexes were then solubilized in the pyrene-SDS micelles. The growth of gold nanoparticles beyond the embryonic stage was subsequently inhibited by the encapsulating SDS and electrophilic pyrene.
\end{abstract}

\section{Introduction}

The current interests in metal nanoparticles (NPs) are driven by their unique chemical and physical properties, which are strongly related to their size and shape. ${ }^{1}$ In particular, gold NP has received intense recent attentions for its applications in biological tagging, ${ }^{2}$ optoelectronics, ${ }^{3}$ and catalysis. ${ }^{4}$ Great efforts have been spent on developing methods for the preparation of gold NP. ${ }^{5-13}$ The stabilizer which protects gold NP plays an important role for controlling the particle size and shape. Organothiols, ${ }^{5-7}$ polymers, ${ }^{8-10}$ and ionic surfactants ${ }^{11-13}$ have been employed as the capping agents in aqueous solution. For ionic surfactants, the usual approach is to match the opposite charges of metal ions and surfactant ions and to perform the chemical reduction. Therefore, for the standard gold source of $\mathrm{AuCl}_{4}^{-}$, one often employs cationic surfactant such as cetyltrimethylammonium bromide (CTAB). ${ }^{11}$ On the other hand, additives such cyclohexane and acetone that could be solubilized in micellar forms of $\mathrm{CTAB}$ are usually used to promote the formation of $\mathrm{Au}$ nanorods. ${ }^{12,13}$ In contrast to CTAB, sodium dodecyl sulfate (SDS), a typical anionic surfactant, has been only used against cationic metallic source such as $\mathrm{Pd}^{2+}$ in making Pd nanoparticles. ${ }^{14}$ For the preparation of gold NP, SDS

* Author to whom correspondence should be addressed. Fax: +886-2-2366-0954; e-mail: cymou@ntu.edu.tw.

$\dagger$ National Taiwan University.

$\doteqdot$ Tamkang University.

(1) Feldheim, D. L.; Foss, C. A., Jr. Metal Nanoparticles: Synthesis, Characterization and Applications; Marcel Dekker: New York, 2002.

(2) Velev, O. D.; Kaler, E. W. Langmuir 1999, 15, 3693.

(3) Alivisatos, A. P. Science 1996, 271, 933.

(4) Haruta, M. Appl. Catal., A 2001, 222, 427.

(5) Brust, M.; Walker, M.; Bethell, D.; Schiffrin, D. J.; Whyman, R.

J. Chem. Soc., Chem. Commun. 1994, 7, 801.

(6) Weisbecker, C. S.; Merritt, M. V.; Whitesides, G. M. Langmuir 1996, 12,3763 .

(7) Thomas, K. G.; Kamat, P. V. Acc. Chem. Res. 2003, 36, 888.

(8) Longenberger, L.; Mills, G. J. Phys. Chem. 1995, 99, 476

(9) Teranishi, T.; Kiyokawa, I.; Miyake, M. Adv. Mater. 1999, 10, 596

(10) Mossmer, S.; Spatz, J. P.; Moller, M; Aberle, T.; Schmidt. J.; Burchard, W. Macromolecules 2000, 33, 4791.

(11) Jana, N. R.; Gearheart, L.; Murphy, C. J. Langmuir 2001, 17, 6782.

(12) Yu, Y. Y.; Chang, S. S.; Lee, C. L.; Wang, C. R. C. J. Phys. Chem. $B$ 1997, 101, 6661 .

(13) Kim, F.; Song, J. H.; Yang, P. J. Am. Chem. Soc. 2002, 124, 14316 has been used only in physical methods such as laser ablation, ${ }^{15}$ sonication, ${ }^{16}$ and seed growth. ${ }^{17}$ This is due to the weak solubilization ability of negatively charged $\mathrm{AuCl}_{4}{ }^{-}$and the weak protecting ability of gold NP with regard to SDS.

However, small gold NP encapsulated by anionic surfactant such as SDS is much desired in many applications that require the surface charge to be negative. For example, negatively charged gold NPs could be deposited on the specific pattern by electrostatic interactions with positively charged surfaces. ${ }^{18}$ Its elimination of nonspecific binding with negatively charged biological molecules may be exploited in bioanalytical technique such as electrophoresis. In particular, SDS-protected gold NPs could suppress their interaction with the capillary wall in capillary electrophoresis (CE) because of electrostatic repulsion; strong interaction usually results in the adsorption of the analytes on the capillary wall. ${ }^{19,20}$

In this paper, we report that pyrene was used as an additive in the aqueous SDS micellar solution for the synthesis of gold NP. It is found that pyrene has the extraordinary effect not only in decreasing the size of gold NPs but also in narrowing their size distribution. The interesting effect of pyrene on the control of particle size is quite different from other additives reported. ${ }^{12,13} \mathrm{We}$ then show CE can be applied for the characterization of the nanoparticle analytes on the basis of their differences in charge-to-size (or mass) ratios. So, we employ micellar electrokinetic capillary chromatography (MEKC), ${ }^{21,22}$ a powerful hybrid technique of electrophoresis and chro344

14) Lee, C. L.; Wan, C. C.; Wang, Y. Y. Adv. Funct. Mater. 2001, 11

(15) Mafune, F.; Kohno, J. Y.; Takeda, Y.; Kondow, T.; Sawabe, H. J. Phys Chem, B 2001, 105, 5114.

(16) Caruso, R. A.; Ashokkumar, M.; Grieser, F. Langmuir 2002, 18, 7831 .

(17) Kuo, C. H.; Chiang T. F.; Chen L. J.; Huang M. H. Langmuir 2004, 20, 7820.

(18) Vossmeyer, T.; Delonno, E.; Heath, J. R. Angew. Chem., Int. Ed. Engl. 1997, 36, 1081 .

(19) Robson, M. M.; Cikalo, M. G.; Myers, P.; Euerby, M. R.; Bartle, K. D. J. Microcolumn Sep. 1997, 9, 357.

(20) Skoog, D. A.; Holler, F. J.; Nieman, T. A. Principles of Instrumental Analysis, 5th ed.; Saunders College Publishing: Philadelphia, 1998; Chapter 30.

(21) Terabe, S.; Otsuka, K.; Ichikawa, K.; Tsuchiya, A.; Ando, T. Anal. Chem. 1984, 56, 111.

(22) Quirino, J. P.; Terabe, S Science 1998, 282, 465. 
matography, to monitor the size distribution of gold NPs during its synthesis. MEKC exploits the ability of certain ionic surfactants, such as SDS, above critical micelle concentration (CMC) to spontaneously form mobile aggregates or surfactant-encapsulated nanoparticles. In our system, an adequate amount of SDS was added to the reaction solution to stabilize gold NP. The mobility of the aggregates would depend on the charge and the size of the SDS-protected gold NP. Therefore, MEKC seems to be the best choice for studying the growth and size distribution of gold NP.

\section{Experimental Section}

All starting materials and reagents were purchased from Arcos and were used as received. For the preparation of gold NP in the SDS system, $5 \mathrm{~mL}$ of $100 \mathrm{mM}$ SDS solution was used as the mother solution. The preparation involves repeating the same mixing action for many runs. In each run, $0.01 \mathrm{~mL}$ of an aqueous solution of $25 \mathrm{mM} \mathrm{HAuCl} \cdot 3 \mathrm{H}_{2} \mathrm{O}$ was added to the mother solution. Next, $0.03 \mathrm{~mL}$ of an aqueous solution of $13 \mathrm{mM} \mathrm{NaBH}_{4}$ was added. UV-vis absorption spectrum is taken between each run of adding reactants.

For the preparation of gold NP in the pyrene-SDS system, an adequate amount of pyrene was added to $100 \mathrm{mM}$ SDS solution with stirring until the pyrene powder completely dissolved. Then, $5 \mathrm{~mL}$ of the solution was used as the mother solution, followed by the same procedure mentioned above in the SDS system. For electronic absorption and fluorescence measure, the concentration of pyrene in the mother solution was $0.7 \mathrm{mM}$. The absorption spectra were recorded on a Hitachi model U-3010 UV/Vis scanning spectrophotometer. Emission spectra were recorded on a Hitachi 4500 spectrofluorometer. For transmission electron microscopy (TEM) studies, a drop of gold NP solution was placed on a carbon-coated copper grid and the solvent was allowed to evaporate. Specimens were examined on a Hitachi H-7100 transmission electron microscope operating at $100 \mathrm{~K} \mathrm{eV}$.

All the capillary electrophoresis experiments were carried out on the P/ACE-MDQ system (Beckman Coulter Inc., Fullerton, CA) with photodiode-array detector. Before CE experiments, the $100-\mu \mathrm{m}$-i.d. and $365-\mu \mathrm{m}-0 . d$. fused-silica capillary (Polymicro Technologie, Phoenix, AZ) with 10-cm effective length was sequentially rinsed with methanol, $1 \mathrm{M} \mathrm{HCl}$, deionized water, and $1 \mathrm{M} \mathrm{NaOH}$. The separation buffer containing $20 \mathrm{mM}$ SDS was made by titrating $10 \mathrm{mM}$ sodium tetraborate and $10 \mathrm{mM}$ sodium dihydrogen phosphate with concentrated $\mathrm{HCl}$ to $\mathrm{pH} 8.0$. Diluted formamide (1:10 000) was used as neutral marker for electroosmotic flow (EOF) measurement. The sample was hydrodynamically injected into the capillary for $3 \mathrm{~s}$ at $0.5 \mathrm{psi}$. Separations were performed with the anode at sample injection end by applying an electric field strength of $300 \mathrm{~V} / \mathrm{cm}$, and the capillary was maintained at $25{ }^{\circ} \mathrm{C}$. Between each run, the capillary was rinsed with deionized water for $3 \mathrm{~min}$, then with $1 \mathrm{M} \mathrm{NaOH}$ for $4 \mathrm{~min}$, and finally was filled with the running buffer. For polyacryamide (PA) coating experiments, the effective length of the capillary was $20 \mathrm{~cm}$ and the applied electric field was $200 \mathrm{~V} / \mathrm{cm}$. The $\mathrm{pH}$ value of the separation buffer was 8.8 . The detection wavelength was $254 \mathrm{~nm}$. All the gold NPs were prepared in $5 \mathrm{~mL} 20 \mathrm{mM}$ SDS solutions. The concentration of pyrene in the mother solution was $0.12 \mathrm{mM}$. Other reagents were used as those in the preparation of gold NPs.

\section{Results and Discussion}

Figure 1a showed the UV-vis absorption spectra of 5 $\mathrm{mL}$ of $100 \mathrm{mM}$ SDS solution (the mother solution) and after the consecutive 18 runs of adding $\mathrm{HAuCl}_{4}$ and $\mathrm{NaBH}_{4}$. In each run, $0.01 \mathrm{~mL}$ of an aqueous solution of 25 $\mathrm{mM} \mathrm{HAuCl}{ }_{4} \cdot 3 \mathrm{H}_{2} \mathrm{O}$ was added to the mother solution. Next, $0.03 \mathrm{~mL}$ of an aqueous solution of $13 \mathrm{mM} \mathrm{NaBH} 4$ was added. UV-vis absorption spectrum is taken between each run of adding reactants. In Figure 1a, the intensity for the $520-\mathrm{nm}$ surface plasmon resonance (SPR) band gradually increases as the reaction proceeds (only runs 1-9 and the last one are shown). Similar results may be
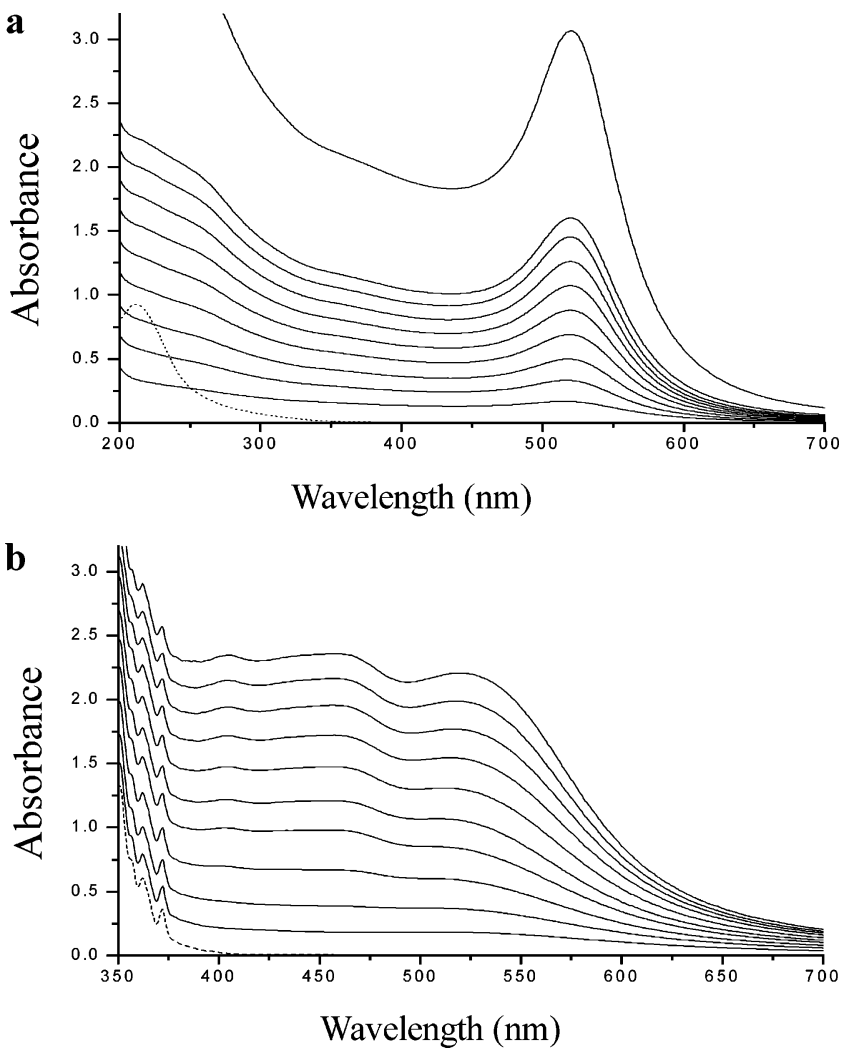

Figure 1. Absorption spectra of solutions of SDS without (a) and with (b) pyrene after the consecutive runs. In a, the dash line was obtained before the first run reduction and only runs 1-9 and 18 are shown. In b, the dash line represents the absorption of pyrene in solution and runs 1-10 are shown.

obtained by changing SDS concentration from $100 \mathrm{mM}$ to $20 \mathrm{mM}$ in the preparation of gold NP. However, if the SDS concentration was below CMC ( $8 \mathrm{mM})$, one would instead obtain gold precipitates. When pyrene was added to the aqueous $100 \mathrm{mM}$ SDS solutions, the same procedure of mixing was followed. Figure $1 \mathrm{~b}$ shows the absorption spectra of each run in which the concentration of pyrene was $0.7 \mathrm{mM}$ in the mother solution. In Figure 1b, the SPR absorption bands are much broader indicating a very small size gradually appearing as the $\mathrm{HAuCl}_{4}$ solution was gradually added to the mother solution. On the other hand, two unusual bands at 410 and $470 \mathrm{~nm}$ appear.

Figure 2a and Figure 2b shows TEM images of gold NPs for the samples in Figure 1a and Figure 1b, respectively. The gold NPs obtained in the SDS solution have an average diameter of $14.1 \mathrm{~nm}$ (Figure 2a), but the average diameter of the gold NPs in the presence of pyrene appears to be $3.7 \mathrm{~nm}$ (Figure 2b). Unexpectedly, the additive pyrene shows an unusual ability to decrease the size of gold NP. It was found that the effect depends on the concentration of pyrene in SDS micellar solution. Figure 2c and Figure 2d shows the particle size distributions obtained from the TEM analysis of gold NPs for the samples in Figure 2a and Figure 2b, respectively. From the size distribution in Figure 2d, it is prominent that pyrene has the extraordinary effect in narrowing the size distribution of gold NPs besides decreasing the size of gold NPs.

The fluorescence spectrum (Figure 3), with excitation wavelength of $340 \mathrm{~nm}$ of the gold NP prepared in the pyrene-SDS solution showed the characteristic pyrene emission band between 380 and $460 \mathrm{~nm}$ and the excimer band from 430 to $600 \mathrm{~nm}$. The fluorescence intensities of the two bands are about equal for the SDS/pyrene/gold 
a
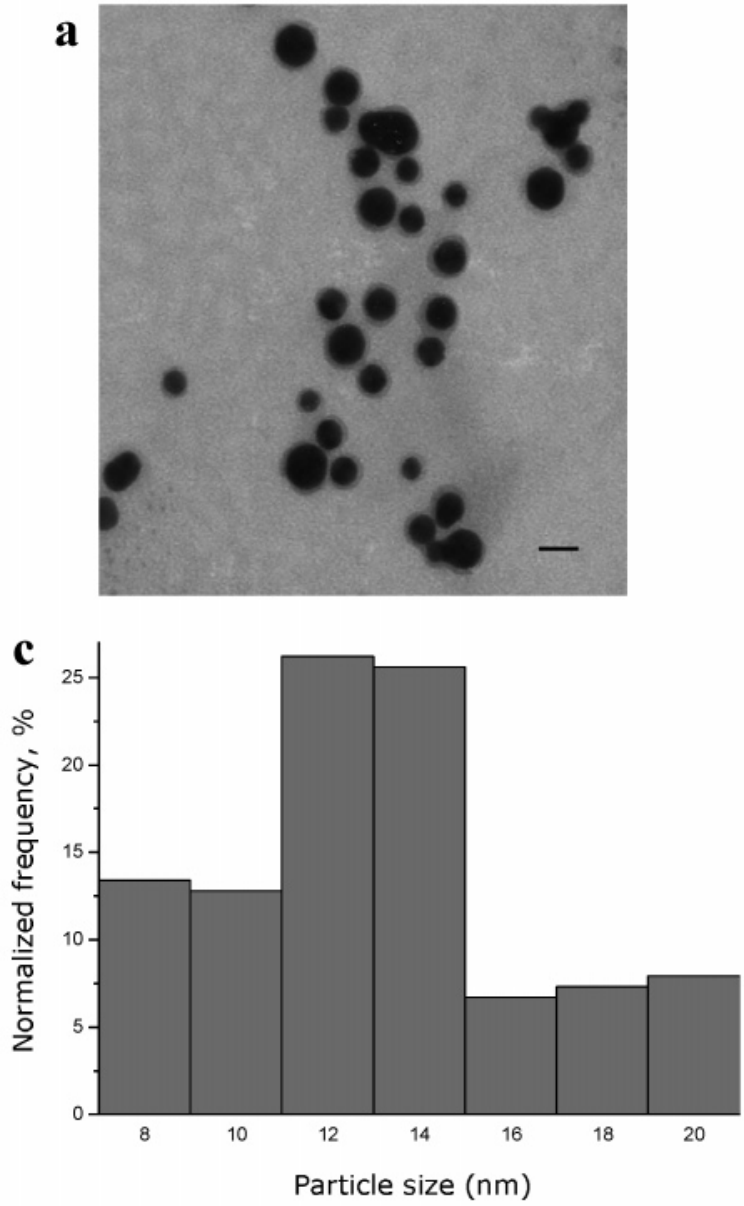

b
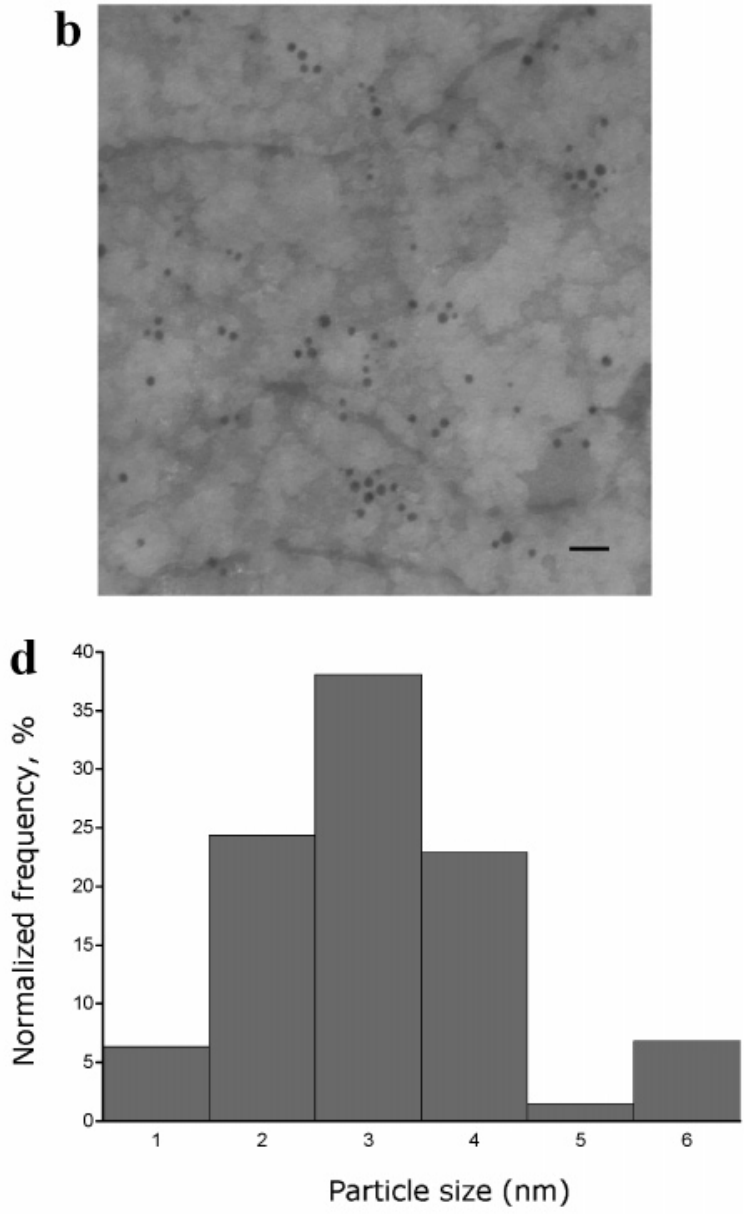

Figure 2. (a) and (b) are TEM images of gold NPs prepared in the reaction solutions in Figure 1a and Figure 1b, respectively. The scale bars indicate $25 \mathrm{~nm}$. (c) and (d) are the particle size histograms from the analysis of the gold NPs in a and b, respectively.

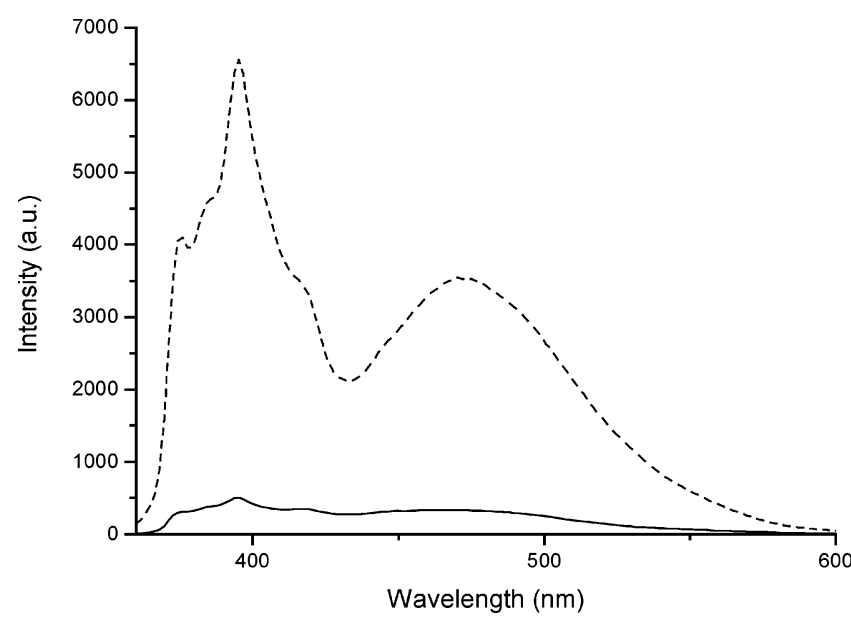

Figure 3. Fluorescence spectra of the reaction solution in Figure 1b before (dash line) and after (solid line) gold NPs are formed.

NP assembly system (the sample of Figure 1b). However, when comparing with the emission spectrum for the same SDS/pyrene/ $\mathrm{NaBH}_{4}$ concentration but without $\mathrm{HAuCl}_{4}$ (dash line in Figure 3), the emission of the sample with gold NPs is strongly quenched. We propose the quenching results from electron transfer from gold NP to pyrene. Furthermore, the $I_{1} / I_{3}$ ratio of the pyrene fluorescence fine structure is a good indicator of the polarity of the local environment. ${ }^{23} \mathrm{We}$ obtain a value of $I_{1} / I_{3}=0.6$ indicating no water penetration into the region of residence of pyrene. This shows that the pyrene molecules are intimately in contact with the SDS protected gold surface where the environment is hydrophobic.

We then examine the electrophoretic behaviors of the SDS protected gold NPs. Figure 4a shows the electropherograms, detected at 254 and $520 \mathrm{~nm}$ wavelength, respectively, of the reaction mixture in the pyrene-SDS solution. Since the SPR absorption of gold NPs is at 520 $\mathrm{nm}$, the peak at about $3.3 \mathrm{~min}$ is assigned to the SDS protected gold NPs and the other peak at $4 \mathrm{~min}$ is assigned to the pyrene-solubilized SDS micelle. The shorter elution time of the neutral marker at around 1 min indicates a high EOF velocity toward the negative electrode. The negatively charged pyrene-solubilized SDS micelles having the electrophoretic flow toward the positive electrode are also carried toward the negative electrode by EOF, but at a lower velocity. The fact that gold NP elutes after neutral marker shows the formation of the negatively charged SDS-capped gold NP. The gold NP possesses a lower charge-to-size ratio than the bare micelle. To probe the embryonic gold NP and its size distribution, electrophoretic analyses of the reaction solutions between each mixing run are performed and these results are shown in Figure $4 \mathrm{~b}$. It can be clearly seen that shorter migration time of gold NP was obtained at increasing runs from the beginning of the synthesis reaction. Their electrophoretic mobility decreased from 3.60 to $3.49 \times 10^{-4} \mathrm{~cm}^{2} \mathrm{~V}^{-1} \mathrm{~s}^{-1}$. These results indicate a decrease in the charge-to-size ratio of the growing gold NP in the embryonic period. The differences in migration time of gold NP become negligible

(23) Kalyanasundaram, K.; Thomas, J. K. J. Am. Chem. Soc. 1977 99,2039 
a
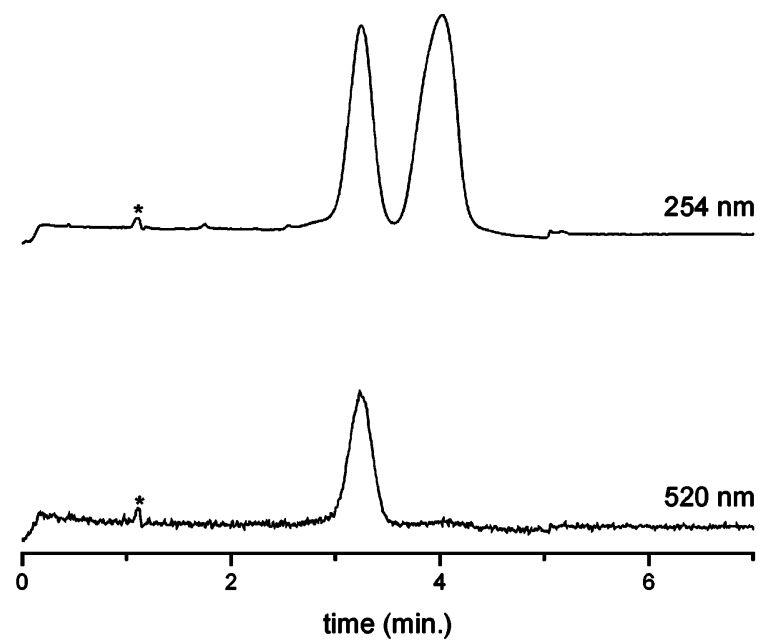

b

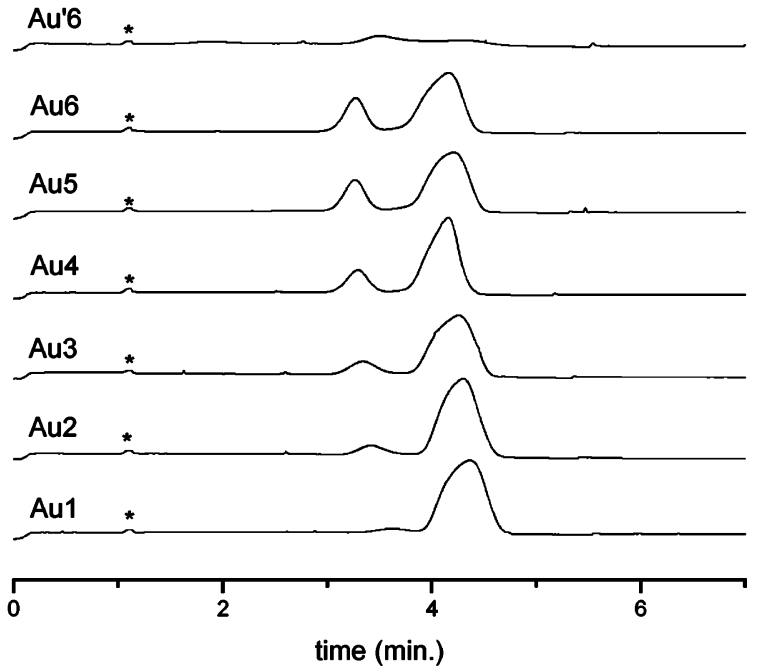

Figure 4. (a) Electropherograms of the SDS solution with pyrene after 10 runs. (b) After consecutive $1-6$ runs (Au1-6) and without pyrene after 6 runs (Au'6). The peak labeled with an asterisk $(*)$ is the neutral marker.

after the sixth run. It is apparent that the peaks of gold NP in Figure 4b (Au1-Au6) always maintain symmetrical shapes without broadening at increasing runs, but a very broad peak $\left(\mathrm{Au}^{\prime} 6\right)$ is observed for the gold NP sample prepared in the reaction solution without the addition of pyrene. The results clearly show that pyrene has the extraordinary effect on decreasing the size and narrowing the dispersity of gold NP (also supported by those results shown in Figure 2). MEKC provided a method of studying the size and dispersity of gold NP. ${ }^{24}$ From the comparison of MEKC electropherograms and size distributions obtained from the TEM image in Figure 2d, we estimate that the resolution limit of MEKC is about $1 \mathrm{~nm}$. For example, the mean size of gold NP in Au1 of Figure 4b, where the gold NP can be just barely resolved, is $1 \mathrm{~nm}$. Also, the peak width would reflect size distribution. However, this requires careful calibration in future work.

On closer inspection of Figure $4 \mathrm{~b}$, the shapes of the peaks at about 4 min seemed asymmetric. There could be some unresolved peaks enveloped in the pyrene peak. To resolve these peaks, PA coating on the inner wall of the capillary column was employed to eliminate the negative charge on the surface of the capillary. Better resolution of electrophoretic separation could be expected because of

(24) Templeton, A. C.; Cliffel, D. E.; Murray, R. W. J. Am. Chem. Soc. 1999, 121, 7081. $\mathbf{a}$

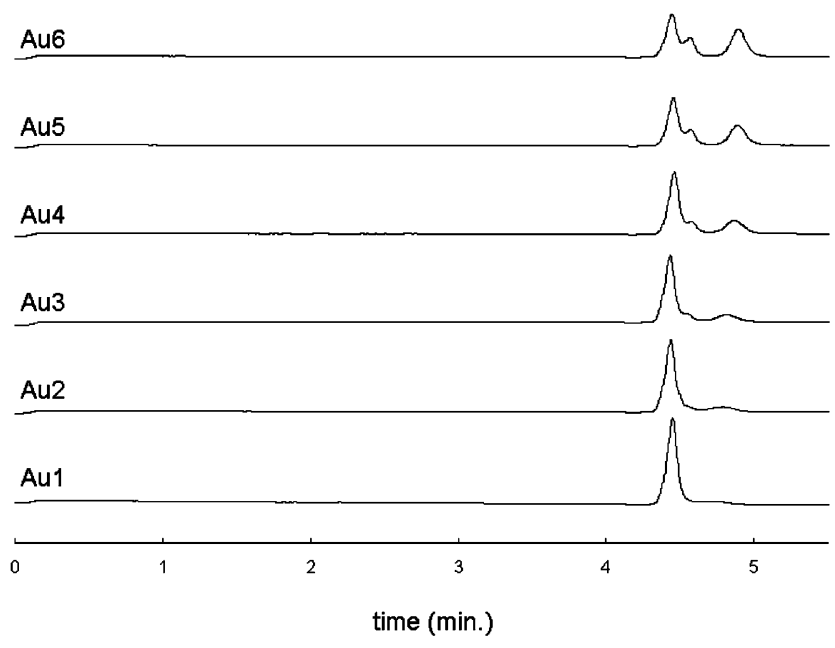

b

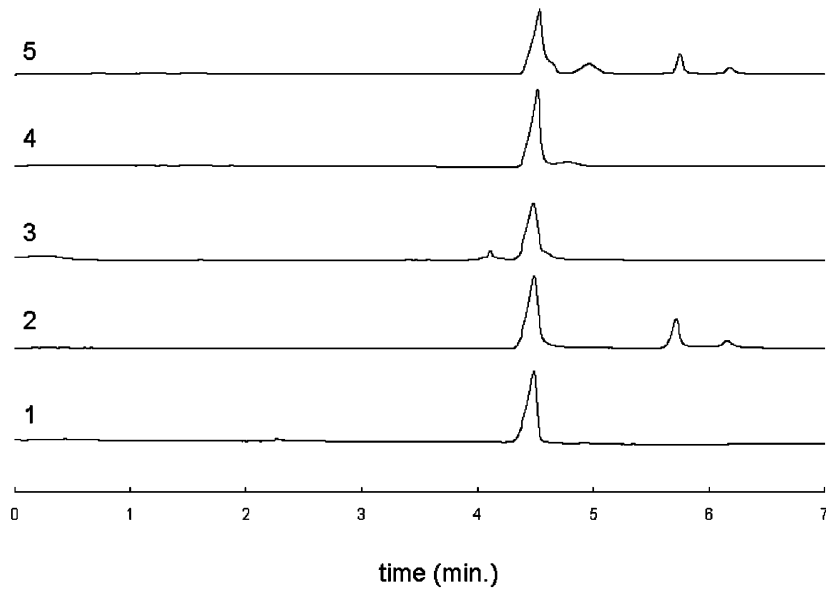

Figure 5. Electropherograms of the solution of SDS with pyrene (a) after consecutive 1-6 runs (Au1-6). (b) Without $\mathrm{NaBH}_{4}$; before any addition of $\mathrm{HAuCl}_{4}$ (line 1), 5 min after adding $0.06 \mathrm{~mL} \mathrm{HAuCl}_{4}$ (line 2), 2 days after (line 3), seed NP of Au1 (line 4) and 25 min after adding $0.05 \mathrm{~mL} \mathrm{HAuCl}_{4}$ to the sample in line 4 (line 5). Electrophoresis conditions: PA-coated capillary.

the suppressions of both EOF and possible solute-inner wall interactions. Figure 5a showed electrophoretic analyses of the reaction solutions between each run in PAcoated capillary. Because the separation of the sample depends on the electrophoretic flow, the elution order in Figure $5 \mathrm{a}$ is opposite to that in Figure $4 \mathrm{~b}$. The peak that was unresolved in the bare capillary gradually appeared at increasing runs and eluted just after pyrene. The appearance of the new peak suggests that pyrene could undergo some chemical reaction with $\mathrm{Au}$ (III) during the preparation of the gold NP.

If only $\mathrm{HAuCl}_{4}$ and pyrene were added to the SDS solution, without the addition of $\mathrm{NaBH}_{4}$, two new bands in the UV-vis absorption spectrum of the reaction solution were observed 2 days after mixing. One peak ranging from 400 to $500 \mathrm{~nm}$ was very broad (similar to that in Figure $1 \mathrm{~b}$ ), and the other peak had the maximum absorption near $550 \mathrm{~nm}$. Figure 5b-2 and Figure 5b-3 show, respectively, the electropherograms of the pyrene-SDS solution obtained 5 min after and 2 days after mixing with $\mathrm{HAuCl}_{4}$. In Figure 5b-2, in addition to the pyrene peak as in Figure $5 \mathrm{~b}-1$, the two peaks around 6 min elution times are due to solution species of $\mathrm{Au}(\mathrm{III})$. In Figure 5b-3, there are a major peak exhibiting a small shoulder and a minor peak 
eluted at shorter time. The unsolubilized solution $\mathrm{Au}$ (III) species at higher elution time disappear, suggesting that the new $\mathrm{Au}$ (III) species produced by the interaction with pyrene are solubilized in the SDS micelles. Without the reducing agent $\mathrm{NaBH}_{4}$, the changes were obviously much slower than those, which occurred in several minutes, in our original preparation method (as in Figure 4). To show that gold NP accelerates the reduction process, embryonic gold particles (sample Au1 in Figure 4a) were first formed in pyrene-SDS solution (electropherogram shown in Figure 5b-4) and then $\mathrm{HAuCl}_{4}$ was added to the above solution. Figure $5 \mathrm{~b}-5$ showed that the Au NP peak appeared quickly in a few minutes, indicating that gold NP could accelerate the interaction of $\mathrm{Au}$ (III) with pyrene. It seems pyrene could reduce $\mathrm{Au}(\mathrm{III})$ to $\mathrm{Au}(0)$ in the presence of gold NP. Similar reduction was also reported in the preparation of gold NP in the aqueous poly(ethylene glycols) solution without any reducing agents. ${ }^{8}$

On the basis of the above results, we can sketch a picture accounting for the formation of gold NPs in SDS and pyrene-SDS micelle solutions. When $\mathrm{HAuCl}_{4}$ was added to the aqueous SDS solution, it dissociated into $\left[\mathrm{AuCl}_{4}{ }^{-}\right]$ and $\left[\mathrm{AuCl}_{3}\left(\mathrm{H}_{2} \mathrm{O}\right)\right]$ species. The latter subsequently dissociated into proton and $\left[\mathrm{AuCl}_{3}(\mathrm{OH})^{-}\right] . .^{25}$ The $\left[\mathrm{AuCl}_{4}{ }^{-}\right]$and $\left[\mathrm{AuCl}_{3}(\mathrm{OH})^{-}\right]$complexes were observed at longer elution times (5.76 and $6.19 \mathrm{~min}$ ) in Figure 5b-2. The neutral $\left[\mathrm{AuCl}_{3}\left(\mathrm{H}_{2} \mathrm{O}\right)\right]$ complex existed in low abundance, so it could not be detected in the PA-coated capillary. Compared with the shorter elution time of the pyrene-solubilized SDS micelles, both $\left[\mathrm{AuCl}_{4}^{-}\right]$and $\left[\mathrm{AuCl}_{3}(\mathrm{OH})^{-}\right]$were unsolubilized in the aqueous phase because of their negative charge. Gold NP rapidly forms when the $\left[\mathrm{AuCl}_{4}{ }^{-}\right.$, $\left[\mathrm{AuCl}_{3}(\mathrm{OH})^{-}\right]$, and $\mathrm{NaBH}_{4}$ react in the aqueous phase. Subsequent capping by the SDS molecules quenches the growth. If gold NP forms too fast for the surfactants nearby to trap during the period of nucleation, then the particle would grow to a larger size. The step-by-step addition of $\mathrm{HAuCl}_{4}$ also slows down somewhat the formation rate of gold NP to let the weak interacting surfactant SDS to encapsulate the NPs.

These $\mathrm{Au}(\mathrm{III})$ complexes are reduced by aryl alcohol ${ }^{26}$ and poly(ethylene glycols) in the formation of gold NP at room temperature. ${ }^{8}$ So, it is possible that pyrene could be oxidized by the $\mathrm{Au}(\mathrm{III})$ slowly in the pyrene-SDS micelle solution before the addition of $\mathrm{NaBH}_{4}$. The oxidized product of pyrene appeared with the concomitant consumption of these gold complexes (no residual $\mathrm{HAuCl}_{4}$ was observed). The absorption peak from 400 to $500 \mathrm{~nm}$ in the UV-vis (Figure 1b) can be attributed to oxidation product of pyrene because its intensity apparently increased with the amount of pyrene added in our pyrene993.

(25) Moodley, K. G.; Nicol, M. J. J. Chem. Soc., Dalton Trans. 1977, (26) Pal, B.; Sen, P. K.; Sen Gupta, K. K. J. Phys. Org. Chem. 2001, 14,284 .
SDS system. The absorption peak was also observed 2 days after only $\mathrm{HAuCl}_{4}$ and pyrene were added to the SDS solution, without the addition of $\mathrm{NaBH}_{4}$. The other absorption peak at $550 \mathrm{~nm}$ in the same solution could be contributed to the reduced Au complexes. Because these reduced $\mathrm{Au}$ complexes have shorter retention times (Figure 5b-3) than those of free ion species (Figure 5b-2), it suggests that all the reduced Au complexes were solubilized in the SDS micelles finally. Thereafter, the reduced $\mathrm{Au}$ complexes evenly distributed in the micelles could interact with the added $\mathrm{NaBH}_{4}$ in the preparation of gold NPs to result in very small gold NPs which were easily capped and stabilized by the outer SDS. The reduction of the Au complexes around the gold NP may be assisted by electron transfer through the gold NP. The electrophilic pyrene and its oxidized derivative may be taking away electrons to inhibit further reduction reaction. The inhibition of growth allows continuous nucleation to form gold NP of really small size. The extraordinary effects of pyrene on both decreasing the size and narrowing the dispersity of the gold NP are due to the chemical reaction of pyrene with the Au complexes and subsequent inhibition of the growth of gold NPs at the embryonic stage. Peculiarly, other aromatics such as naphthalene and anthracene do not exhibit the same size-reduction effect as pyrene does. The difference may be due to the different reduction potentials.

\section{Conclusions}

In conclusion, we have successfully synthesized gold NPs in SDS micellar solution by chemical reduction using $\mathrm{NaBH}_{4}$. The addition of pyrene in SDS micellar solution results in the formation of gold NPs in smaller size and narrower distribution. We have demonstrated MEKC technique can be applied to analyze the size distribution of SDS protected gold NPs. The combination of pyrene and SDS provides an excellent method for controlling the size and dispersity of negatively charged monolayer protected NPs in aqueous solution. We not only find a method in controlling the size in Au@SDS, which is very difficult to do because of the same charge of SDS and $\mathrm{AuCl}_{4}^{-}$, but we also studied the mechanism of the formation process of gold NP. This understanding would be useful in developing general strategies for size control of metallic nanoparticles in the future. With our method, other anionic stabilizers ${ }^{27}$ for nanocluster formation may also be developed.

Acknowledgment. This work was supported by a grant from the National Science Council of Taiwan through the National Nano-Initiative program (NSC-93-2120-M002-003).

\section{LA051252M}

(27) Ozkar, S.; Finke, R. G. J. Am. Chem. Soc. 2002, 124, 5796. 